\title{
Dimensional Analysis in the Machining of Housing Components with Cast Holes
}

\author{
V. N. Ashikhmin and S. S. Kugaevskii \\ Yeltsin Ural Federal University, Yekaterinburg \\ e-mail:cad-cam@mail.ru
}

\begin{abstract}
When cast housing components are machined, the displacement of the hole axis in reaming must be taken into account, because it produces pronounced nonuniformity of the margin. Means of reducing the machining error are analyzed.
\end{abstract}

Keywords: machining, casting holes, dimensional analysis, margin, displacement

DOI: $10.3103 / \mathrm{S} 1068798 X 13090049$

The reaming of holes in cast housings with slight displacement of the hole axis is not difficult if a single setup is used. The margin removed in the two successive operations is uniform in this case, as is evident in Fig. 1. In the first operation, a hole of radius $R_{i-1}$ is made. (The rated hole radius is $R_{i}$.) The margin $Z_{i}$ will then be the same over the whole diameter. However, with two successive operations in different setups, the margin is nonuniform, on account of the displacement of the hole axis. This problem is particularly apparent in the machining of cast housing blanks.

Consider the influence of the displacement $\rho_{i}$ of the hole axis (Fig. 2) in successive operations with a nonuniform margin $\left(Z_{i}^{\prime \prime}-Z_{i}^{\prime}\right)$. In the initial blank, prior to the first operation, the hole radius is $R_{i-1}$. The radius $R_{i}$ will be obtained in the first machining operation, with displacement $\rho_{i}$ of the hole axis in the blank.

The theoretical margin with no displacement of the hole axis is $Z_{i}$. In Fig. 2, the dashed curve shows the position of the surface corresponding to no displacement of the hole axis in the next operation.

Displacement of the hole axis in either direction is equiprobable (Figs. 2a and 2b). Therefore, the rated displacement is assumed to be zero. The change in the margin with displacement $\rho_{i}$ in the two possible directions will be $Z_{i}^{\prime}=Z_{i}-\rho_{i} ; Z_{i}^{\prime \prime}=Z_{i}+\rho_{i}$. Correspondingly, the total nonuniformity of the margin is $2 \rho_{i}=$ $Z_{i}^{\prime \prime}-Z_{i}^{\prime}$.

If we disregard the displacement of the hole axis and it is larger than the margin $Z_{i}$, traces of the initial blank's cast surface will be visible on one side of the machined hole.

The influence of the displacement $\rho_{i}$ on the margin $Z_{i}$ may be established by means of a dimensional chain
(Fig. 3). The right-facing arrows indicate increase in the corresponding quantity, while the left-facing arrows indicate decrease.

With no displacement of the hole axis, the minimum margin $Z_{i \min }$ is the same in both directions

$$
Z_{i \min }=R_{i \min }-R_{i-1 \max } .
$$

With displacement of the hole axis, we conclude from the dimensional chain that

$$
Z_{i \min }=R_{i \min }-R_{i-1 \text { max }}-\rho_{i \max } .
$$

The radius $R_{i \min }$ remains constant and corresponds to the minimum value of the structural dimensions (in one-time machining). The margin $Z_{i \min }$ also remains constant and is assumed to correspond to the standard for the given type of treatment. The variable is $\rho_{i}$, which depends primarily on the selection of the base in machining and the setup of the dimensions for the initial blank. Therefore, in accordance with

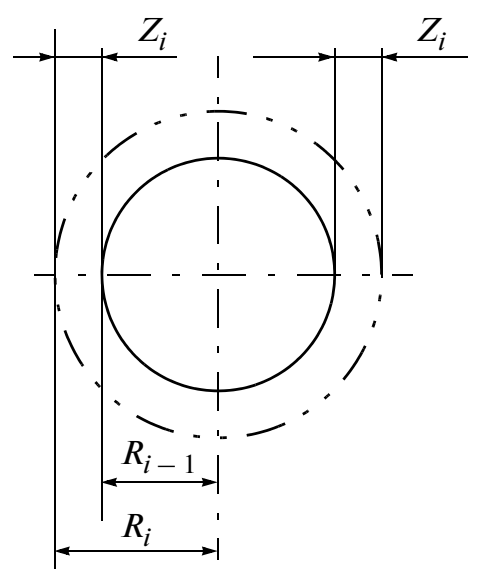

Fig. 1. Machining with a uniform margin. 
(a)

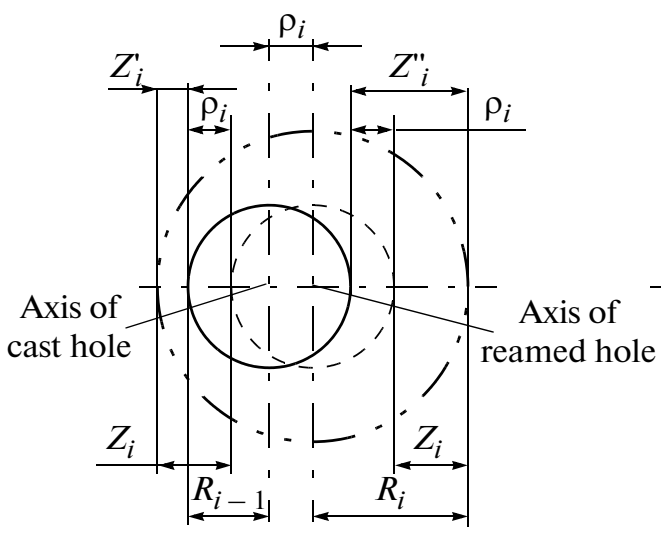

(b)

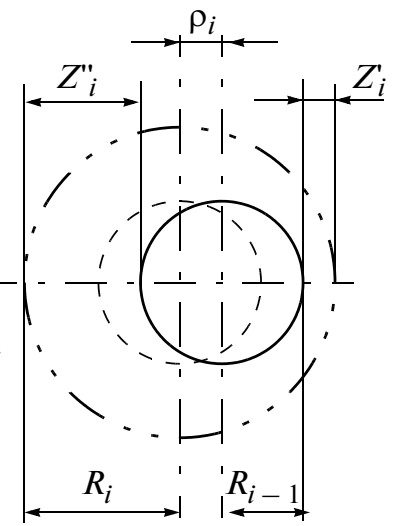

Fig. 2. Influence of displacement of the hole axis on the nonuniformity of the margin.

Eq. (1), $R_{i-1 \max }$ must decrease with increase in $\rho_{i \max }$. In other words, with constant margin, the rated radius of the cast hole must be reduced. That increases the rated margin in machining. If the displacement of the hole axis is not taken into account in selecting the margin, we may find that the displacement exceeds the margin and that traces of the cast surface are visible on one side of the machined hole.

We now consider the influence of displacement of the hole axis on the nonuniformity of the margin for a specific example (Fig. 4). Three planes are used for basing in the broaching operation. The calculations are conducted for the $Z$ direction. We specify $K_{1}=$ $40 \pm 0.31 \mathrm{~mm} ; K_{2}=10_{-0.22} \mathrm{~mm} ; K_{3}=26.5^{+0.15} \mathrm{~mm}$.

The numbers of the surfaces (circled) correspond to refined dimensional analysis on the basis of model graphs of the process [1]. The numbers increase in the direction of the selected axis ( $Z$, in the present case). The generatrix and axis of the machined hole are considered as separate surfaces. Since the first (lower) hole generatrix has a smaller $Z$ coordinate than the hole axis, it is assigned the number 3 , while the hole axis is 4 . For the given component, we machine surfaces 1 and 3 .

In constructing a dimensional representation and graphs, the numbers of the surfaces in the final part are denoted by multiples of 10 . The numbering of the intermediate surfaces and the surfaces of the initial blank changes by one each time that the next intermediate margin is added to the surface of the final part or the already machined surface or each time that the axis

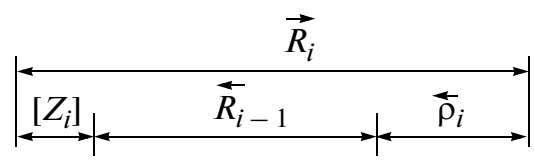

Fig. 3. Dimensional chain for the displacement of the hole axis. is displaced. In other words, with increase in $Z$, the number of the surface will be larger. In this example, the roughness of all the machined surfaces is $R a=$ $6.3 \mu \mathrm{m}$. The dimensional precision after machining corresponds to class 12-14. Therefore, one-time machining of each surface is sufficient. In that case, the base in the initial blank (with the addition of margin $Z_{1}$ ) will be assigned the number 9 , while the lower generatrix of the hole in the initial blank with margin $Z_{2}$ will be 31 . The displacement of the hole axis after machining corresponds to the rated value (zero). Therefore, the hole axis in the initial blank may be higher or lower than in the final part (Fig. 4). Consider the most unfavorable case, when the displacement of the hole axis in machining reduces the margin $Z_{2}$ (Fig. 2a). That means that the hole axis in the initial blank is surface 39.

In the initial version of the process, the dimensions $L_{1}, L_{2}, L_{3}$ (Fig. 5) of the initial blank are represented by the design dimensions $K_{1}, K_{2}, K_{3}$ (Fig. 4). The minimal margins are $Z_{1 \min }=1.1 \mathrm{~mm} ; Z_{2 \min }=1.3 \mathrm{~mm}$.

The tolerances are $I T_{L 1}=2 \mathrm{~mm} ; I T_{L 2}=1.4 \mathrm{~mm}$; $I T_{L 3}=1.0 \mathrm{~mm}$.

We now formulate the dimensional diagram for a simple example, with two operations (Fig. 5): milling 05 (machining of the base); and broaching 10 (hole machining).

In the first case, the base in the first operation is the surface of the plate that is not being machined; the base in the second operation is the milled foundation plate.

The following rules are used to verify the dimensional diagram. The number of vertical lines must be one greater than the number of technological dimensions $L$. (The dimensions of the initial blank are included here.) The number of final dimensions in the dimensional chains (equal to the sum of the number of design dimensions, margins, and displacements) must 


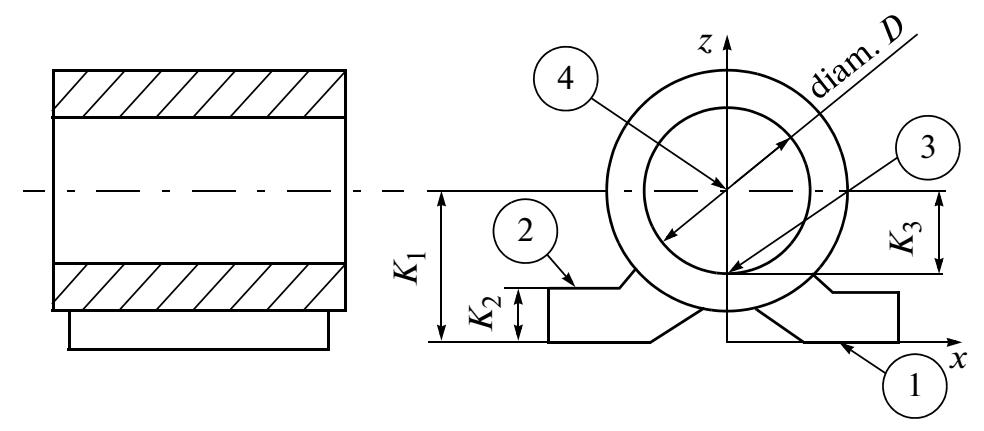

Fig. 4. Sketch of part.

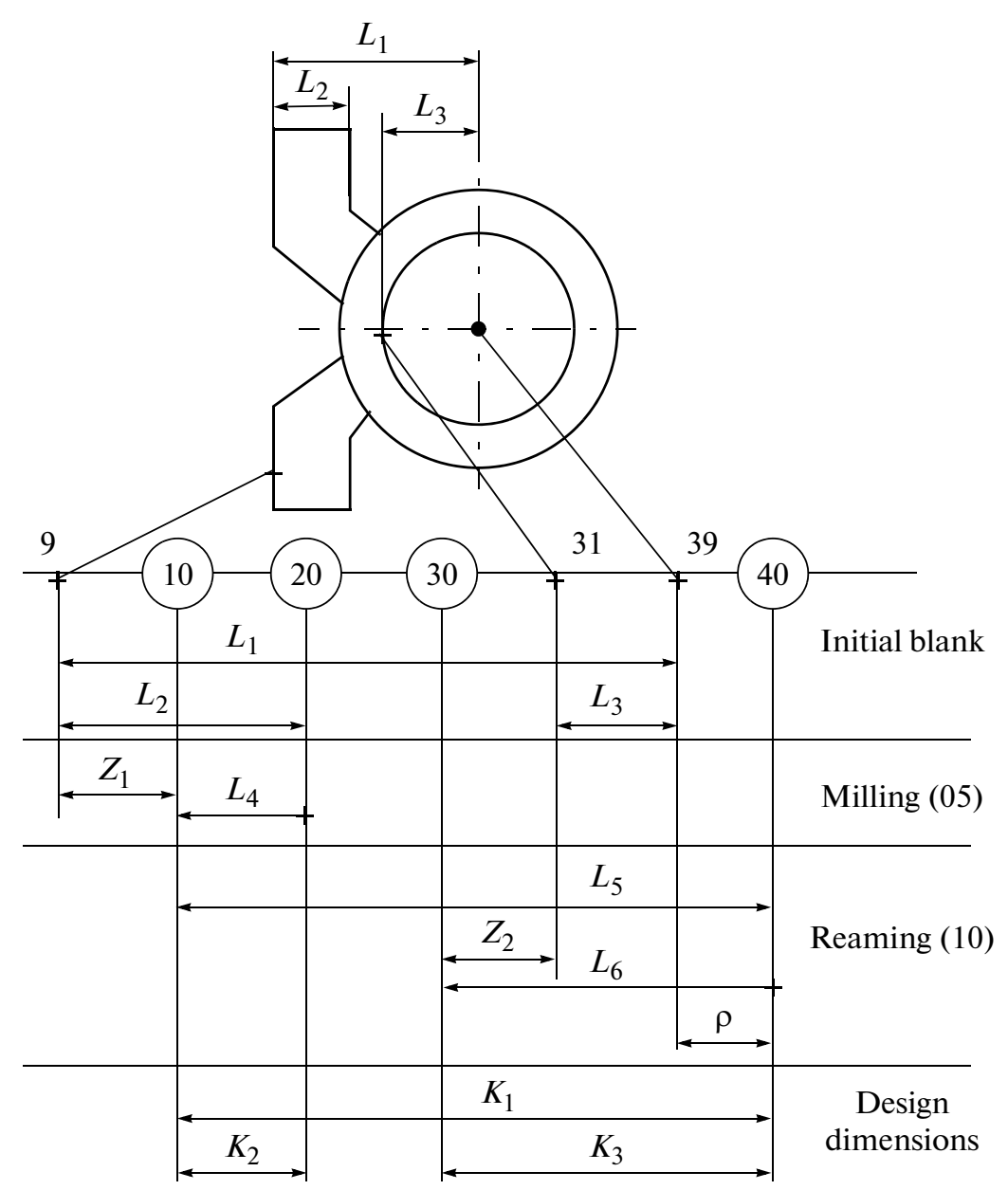

Fig. 5. Dimensional diagram.

be equal to the number of technological dimensions (elements in the technological dimensional chains). These requirements are satisfied in Fig. 5.

The graphs of the technological processes are plotted on the basis of the rules in [2]. The initial graph, containing all the final elements, is shown in Fig. 6; its derivative is shown in Fig. 7. Table 1 presents the components and equations of the dimensional chains obtained on the basis of refined dimensional analysis [2].

The chain with final element $Z_{2}$, in which the displacement $\rho$ affects the margin, is the most difficult to analyze.

Next, by superimposing the derivative graph on the initial graph, we obtain the composite graph in Fig. 8, 


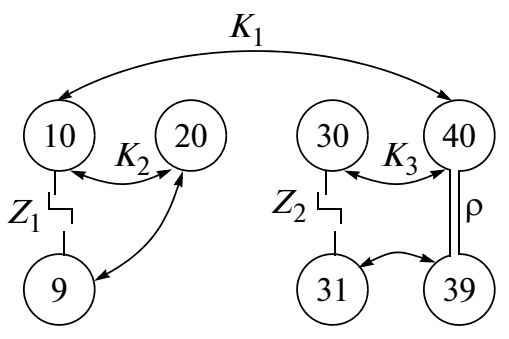

Fig. 6. Initial graph.

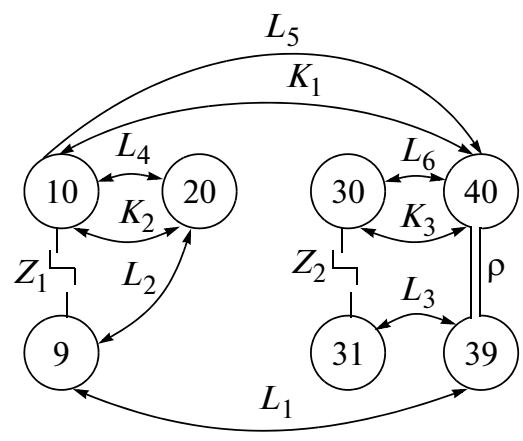

Fig. 8. Composite graph.

which is the mathematical model of all the dimensional relations of the processes. This graph may expediently be used in analyzing how the displacement of the hole axis affects the margin in broaching. To this end, we use the particular graph in Fig. 9, where the displacement $\rho$ is a component, and the margin $Z_{2}$ is the final element. The equation of that chain takes the form

$$
-Z_{2}+L_{6}-\rho-L_{3}=0 .
$$

In that case, two rows are added to the calculation table (Table 2). The first row determines the margin for the displacement of the hole axis when it is the final element. Since the rated displacement of the hole axis is zero, the maximum and minimum displacement will be equal to half the tolerance with positive and negative signs, respectively. The second row shows the

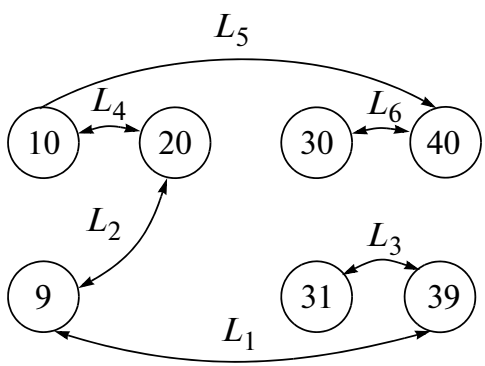

Fig. 7. Derivative graph.

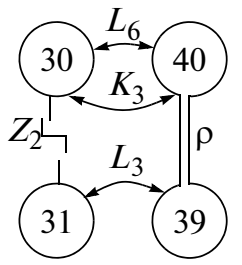

Fig. 9. Particular graph.

calculation results for the dimensional chain with final element $Z_{2}$ obtained by means of Fig. 9 .

Chains where the final element is a design dimension are calculated in terms of the deviation [2]. Chains where the final element is the margin are calculated in terms of the limiting values. Chains where the final element is the displacement of the hole axis are calculated in terms of the mean values. Therefore, Table 2 presents values of $L_{\max }, L_{\min }$, and $L_{\text {me }}$. In the graph, $L_{\text {me }}$ corresponds to the values of the elements that appear in dimensional chains where the final element is the displacement of the hole axis.

Table 2 presents a sample calculation. In the second highest row, we use only one dependence: the tolerance on the final element, equal to the sum of the tolerances on the components. The tolerance on the displacement is $4.24 \mathrm{~mm}( \pm 2.12 \mathrm{~mm})$.

Table 1. Identification of dimensional chains

\begin{tabular}{c|c|c|l}
\hline Final element & Vertices of initial graph & Sequence of vertices in derivative graph & \multicolumn{1}{|c}{ Equation of dimensional chain } \\
\hline$K_{1}$ & $10 ; 40$ & $10 ; 40$ & $-K_{1}+L_{5}=0$ \\
$K_{2}$ & $10 ; 20$ & $10 ; 20$ & $-K_{2}+L_{4}=0$ \\
$K_{3}$ & $30 ; 40$ & $30 ; 40$ & $-K_{3}+L_{6}=0$ \\
$Z_{1}$ & $9 ; 10$ & $9 ; 20 ; 10$ & $-Z_{1}+L_{2}-L_{4}=0$ \\
$Z_{2}$ & $30 ; 31$ & $30 ; 40 ; 10 ; 20 ; 9 ; 39 ; 31$ & $-Z_{2}+L_{6}-L_{5}+L_{4}-L_{2}+L_{1}-L_{3}=0$ \\
$\rho$ & $39 ; 40$ & $39: 9 ; 20 ; 10 ; 40$ & $-\rho-L_{1}+L_{2}-L_{4}+L_{5}=0$ \\
\hline
\end{tabular}


Table 2. Calculation results for the dimensional chains

\begin{tabular}{l|c|c|c|c|c|c}
\hline \multicolumn{1}{c|}{$\begin{array}{c}\text { Equation } \\
\text { of final element }\end{array}$} & $\begin{array}{c}\text { Element being } \\
\text { determined }\end{array}$ & $\begin{array}{c}\text { Order } \\
\text { of calculation }\end{array}$ & $L_{\mathrm{max}}, \mathrm{mm}$ & $L_{\mathrm{min}}, \mathrm{mm}$ & $L_{\mathrm{av}}, \mathrm{mm}$ & $\begin{array}{c}\text { Operational } \\
\text { dimension, mm }\end{array}$ \\
\hline$K_{1}=L_{5}$ & $L_{5}$ & 1 & 40.31 & 39.69 & 40.00 & $40 \pm 0.31$ \\
$K_{2}=L_{4}$ & $L_{4}$ & 2 & 10.00 & 9.78 & 9.89 & $10_{-0.22}$ \\
$K_{3}=L_{6}$ & $L_{6}$ & 3 & 26.65 & 26.5 & - & $26.5^{+0.15}$ \\
$Z_{1}=L_{2}-L_{4}$ & $L_{2}$ & 4 & 12.5 & 11.1 & 11.8 & $11.8 \pm 0.7$ \\
$\rho=-L_{1}+L_{2}-L_{4}+L_{5}$ & $L_{1}$ & 6 & 42.91 & 40.91 & 41.91 & $41.91 \pm 1.0$ \\
$\rho=-L_{1}+L_{2}-L_{4}+L_{5}$ & $\rho$ & 5 & +2.12 & -2.12 & 0 & $0 \pm 2.12$ \\
$Z_{5}=L_{5}-\rho-L_{5}$ & $L_{3}$ & 7 & 23.08 & 22.08 & - & $22.58 \pm 0.5$ \\
\hline
\end{tabular}

Analysis of the results shows that, with minimum margin $Z_{2 \min }=1.3 \mathrm{~mm}$, the maximum margin is $Z_{2 \max }=6.69 \mathrm{~mm}$, which is more than five times as large. That will impair the machining precision and productivity. The greatest nonuniformity of the margin is associated with large displacement of the hole axis. The use of Fig. 9 in the analysis of the nonuniformity of $Z_{2}$ permits the investigation of the basic displacement $\rho$ of the hole axis. It is evident from the equations that the tolerances $L_{1}$ and $L_{2}$ have the greatest influence on the dimensions of the initial blank.

We consider possible remedies on the basis of the composite graph in Fig. 8. First, we attempt to reduce the number of elements in the dimensional chain for the displacement $\rho$ of the hole axis - for example, by modifying the specification of the initial blank's dimensions. For instance, we may specify dimension $L_{1}$ between surfaces 20 and 39. That leaves Fig. 9 unchanged. The new composite graph is shown in Fig. 10.

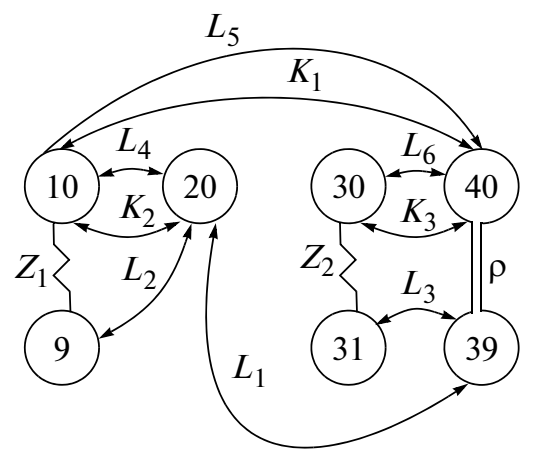

Fig. 10. Modified composite graph.

The new equation takes the form

$$
\rho=-L_{1}-L_{4}+L_{5} .
$$

Since $L_{1}$ falls within the preceding dimensional interval, its tolerance is reduced to $I T_{L 1}=1.8 \mathrm{~mm}$. Then

$$
\begin{gathered}
T \rho=I T_{L 1}+I T_{L 4}+I T_{L 5} \\
=1.8+0.22+0.62=2.64 \mathrm{~mm} .
\end{gathered}
$$

The displacement of the hole axis is reduced by a factor of 1.6. The nonuniformity of the margin, defined as $Z_{2 \max } / Z_{2 \min }$, is reduced from 5.2 to 3.9. Further reduction in the displacement of the hole axis and hence in the nonuniformity of the margin is possible by reducing the tolerances $T L_{4}$ and $T L_{5}$ to the extent possible.

The most effective approach is to use the axis of the cast hole as the rough base. In that case, the displacement of the hole axis is reduced by a factor of 3.5 at least, and the nonuniformity of the margin is halved.

Thus, the proposed dimensional analysis permits the identification of means of improving the machining precision - primarily by changing the basing configuration and the specification of the initial blank's dimensions. In such simulation, options for reducing the displacement $\rho$ are analyzed by means of a composite graph, without changing the particular graph already derived.

\section{REFERENCES}

1. Ashikhmin, V.N. and Zakuraev, V.V., Improving the dimensional analysis of technological processes, Spravochnik, Inzh. Zh., 2009, no. 1(142), pp. 12-15.

2. Ashikhmin, V.N. and Zakuraev, V.V., Razmernyi analiz pri tekhnologicheskom proektirovanii (Dimensional Analysis in Technological Design), Yekaterinburg: GOU VPO UGTU-UPI, 2005.

Translated by Bernard Gilbert 